
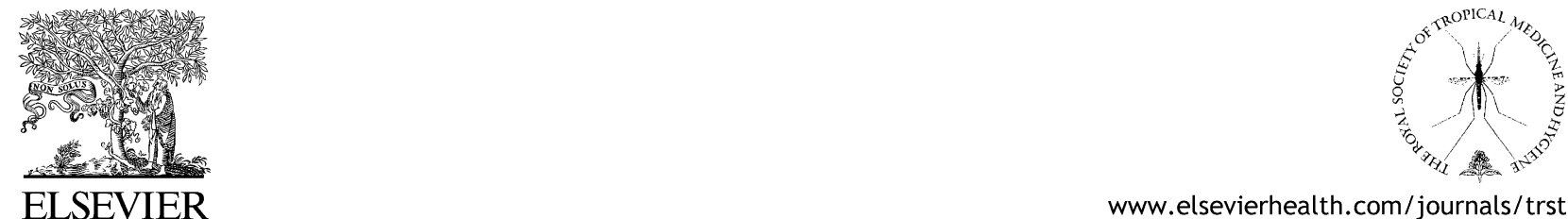

www.elsevierhealth.com/journals/trst

\title{
A prospective study of Plasmodium falciparum multiplicity of infection and morbidity in Tanzanian children
}

\author{
L. Henning ${ }^{a}$, D. Schellenberg ${ }^{b, c}$, T. Smith ${ }^{a}$, D. Henning ${ }^{a}$, P. Alonsoc, \\ M. Tanner ${ }^{\mathrm{a}}$, H. Mshinda ${ }^{\mathrm{b}}$, H.-P. Beck ${ }^{\mathrm{a}}$, I. Felger ${ }^{\mathrm{a}, *}$
}

a Swiss Tropical Institute, Socinstrasse 57, P.O. Box, CH-4002, Basel, Switzerland

b Ifakara Health Research and Development Centre, P.O. Box 53, Ifakara, Tanzania

c Hospital Clinic i Provincial, Villaroel 170, E-08036, Barcelona, Spain

Received 10 November 2003; received in revised form 10 March 2004; accepted 16 March 2004

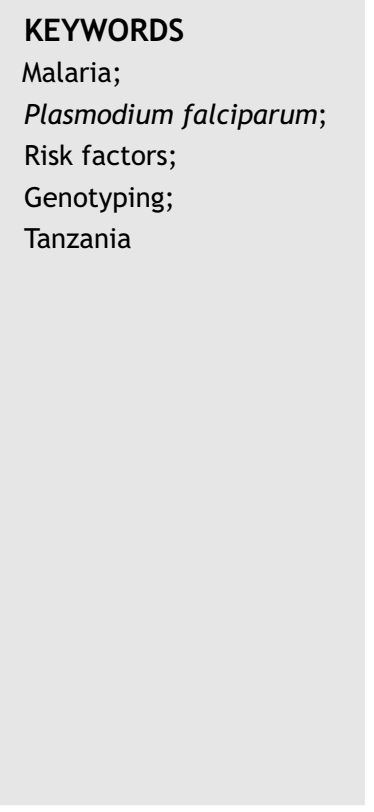

\begin{abstract}
Summary Several studies suggest that in individuals with substantial previous exposure to malaria, co-infection with multiple clones of Plasmodium falciparum can protect against subsequent clinical malaria attacks. Other studies, mainly of individuals with little previous exposure, found the converse relationship. To test whether acquisition of such cross-protection tracks the acquisition of clinical immunity in general, 610 Tanzanian children aged $0-6$ years were enrolled in a nine-month prospective study of the risk of morbidity in relation to parasitological status and merozoite surface protein 2 genotypes on enrolment. Prevalence of parasitaemia and multiplicity of infection increased with age. In the first year of life, the incidence of clinical malaria was almost three times higher in children with parasites at baseline than in those without. In older children, baseline $P$. falciparum infections appeared to protect against both parasitaemic and non-parasitaemic fever episodes. In children aged less than three years, baseline multiple infection tended to be associated with higher prospective risk of clinical malaria than single infection while in children aged more than three years the converse was found, but these effects were not statistically significant. These results provide further evidence that relationships between asymptomatic malaria infections and clinical malaria change with cumulative exposure.

(c) 2004 Royal Society of Tropical Medicine and Hygiene. Published by Elsevier Ltd. All rights reserved.
\end{abstract}

\section{Introduction}

* Corresponding author. Tel.: +41 612848 117; fax: +41612718 654 .

E-mail address: ingrid.felger@unibas.ch (I. Felger).
In areas highly endemic for malaria, many individuals are infected simultaneously with several Plasmodium falciparum clones. The term multiplicity 
of infection (MOI) has been used to describe the number of concurrent infections per carrier. Multiplicity of infection is determined by genotyping highly polymorphic marker genes and reflects the number of different genotypes detected. In areas of high endemicity, multiplicity depends on the age of the host (Ntoumi et al., 1995; Smith et al., 1999a) and on the transmission intensity (Arnot, 1998). In Idete, Tanzania, mean MOI in asymptomatic children aged 3-7 years was five infections per carrier (Beck et al., 1997). In contrast, in children aged less than one year in the same village, mean multiplicity was only two infections per child (Felger et al., 1999).

A number of studies have found relationships between $\mathrm{MOI}$ and the risk of malaria morbidity. Several studies of partially immune children have found that multiple malaria infections are associated with reduced incidence of clinical episodes of $P$. falciparum (Al Yaman et al., 1997a; Beck et al., 1997; Farnert et al., 1999). A study in São Tomé found that the apparent protection extended also to nonmalaria fever episodes (Muller et al., 2001). Other studies, such as those of infants in Idete (Felger et al., 1999; Smith et al., 1999a), of children aged less than three years in western Kenya (Branch et al., 2001), of children in Ghana (Ofosu-Okyere et al., 2001), and of individuals in relatively low transmission settings in the Sudan (Roper et al., 1998) and Mozambique (Mayor et al., 2003) have found that multiple infections were a risk factor for clinical malaria attacks.

To explain these diverse results, we have previously proposed that cross-protection against clinical malaria by concomitant infections may be characteristic of the semi-immune host, in contrast to individuals with less immunity, in whom each additional infection adds to the risk of a clinical attack (Smith et al., 1999b). This implies that the patterns would vary with the degree of malaria endemicity. However, these apparent relationships are also influenced by many other factors, which differ between studies, including survey design and patterns of seeking healthcare (Branch et al., 2001; Muller et al., 2001). To examine how the effect of $\mathrm{MO}$ varies as clinical immunity is acquired, we carried out a cohort study of 610 children in Ifakara, Tanzania, spanning the whole age range (from birth to six years) over which clinical immunity to $P$. falciparum was thought to develop in this area.

We carried out an initial cross-sectional survey of the cohort. Fever episodes were then recorded via a passive case detection system for the following nine months. As for previous studies, we used the highly polymorphic merozoite surface antigen 2 (msp2) as a molecular marker to examine how par- asite genotypes and multiplicity varied with age in the baseline survey. We then tested whether parasite density, multiplicity of infection, or a specific msp2 allelic family of $P$. falciparum were associated with the risk of subsequent febrile episodes and how these relationships varied with age.

\section{Materials and methods}

\subsection{Study site}

The study was conducted in Ifakara town, Kilombero District, Tanzania during the rainy season, starting in March 1998. The Kilombero district is mostly an area of intense and perennial malaria transmission, predominantly $P$. falciparum (Smith et al., 1993) with Anopheles gambiae and $A$. funestus as the two main vectors. Prevalence of $P$. falciparum infections shows no marked seasonality, but mosquito densities, exposure and incidence of clinical malaria episodes in young children vary over the year. A decade ago, the average entomological inoculation rate (EIR) recorded in nearby rural areas was more than 300 infectious bites per person per year (Smith et al., 1993). However, recent field surveys have found much lower levels of malaria transmission, in particular in Ifakara town where an annual EIR of 29 infective bites per person has been estimated (Drakeley et al., 2003). Ifakara town is the site of the St Francis Designated District Hospital (SFDDH), which provides primary health care facilities for the population of the town, as well as acting as a referral hospital for the whole district. Most of Ifakara town has the characteristics of a peri-urban area with a high population density, but with areas of subsistence agriculture interspersed between the houses. Houses are constructed either of traditional-style mud bricks and thatch or of baked bricks with galvanized iron roofs.

\subsection{Cohort recruitment and survey procedures}

A comprehensive list of balozis (ten-cell-leaders who are responsible for approximately 10 households) was used as a sampling frame. A random sample of 610 children ( $50 \%$ male) aged $0-6$ years (mean age 2.9 years), stratified into one-year age groups, was selected by randomly sampling from the list of balozis and recruiting one child at random from each age group within the area covered by each balozi. Children were only recruited to the study after the study was explained to their parents and the latter had signed a letter of consent written 
in Kiswahili. Parents were invited to bring their children to the Maternal and Child Health $(\mathrm{MCH})$ clinic of the SFDDH where two blood slides for microscopic detection of malaria parasite were collected from each child, and a finger-prick blood sample collected in an EDTA microtainer. Research and ethical clearance for this study was obtained from the local (Ifakara) ethical committee and subsequently from the Tanzanian Commission for Science and Technology (UTAFITI), based on the recommendation of the Medical Research Coordination committee of the National Institute for Medical Research (NIMR). Children who were already participating in a health intervention trial were not recruited.

\subsection{Case detection and treatment}

Children enrolled in the study were offered free treatment at SFDDH and were followed-up over nine months until January 1999 by a passive case detection system at the $\mathrm{MCH}$ clinic or the outpatient clinic of SFDDH. All children reporting sick were seen by a project clinical officer. A fingerprick blood sample was collected in an EDTA microtainer and a blood slide was prepared. Those with slide-positive malaria were given a standard treatment of chloroquine $(25 \mathrm{mg} / \mathrm{kg})$ for three days or sulfadoxine-pyrimethamine, in line with national policy. Those with a packed cell volume $<25 \%$ received iron supplementation $(6 \mathrm{mg} / \mathrm{kg}$ daily) for two weeks and a treatment dose of antimalarial, according to hospital guidelines.

For the purposes of the present analysis, febrile episodes were defined as those with axillary temperatures $>37.4{ }^{\circ} \mathrm{C}$ and clinical malaria episodes were febrile episodes subsequently confirmed by microscopy to be $P$. falciparum-positive. The small sample size precluded any formal definition of clinical malaria cases using parasitaemia cut-offs $>0$.

\subsection{Blood slide analysis}

Thick and thin blood films were air-dried and stained with Giemsa's stain for microscopy. Parasite density was assessed by counting the number of asexual stage parasites per 200 leucocytes. The number of parasites relative to the leucocyte count was converted to parasites per microlitre of blood by assuming a leucocyte count of $8000 / \mu l$. Standard quality control procedures established in the Ifakara laboratory were applied (Alonso et al., 1994).

\subsection{Genotyping}

Blood pellets were transferred to IsoCode ${ }^{T M}$ Stix PCR template preparation dipstick (Schleicher and Schuell, Dassel, Germany) for storage, transport and DNA isolation (Henning et al., 1999). All parasites in microscopically-positive children, both in the baseline surveys and from sick children, were genotyped by PCR-RFLP using the msp2 of $P$. falciparum as a marker gene. In addition, a $20 \%$ random sample of the microscopy-negative samples from the baseline survey was also analysed by PCR. PCR-RFLP genotyping of msp2 was performed as described by Felger and Beck (2002). The increased sensitivity of detection by PCR resulted in a number of previously undetected infections. By assuming the positivity rates measured for this $20 \%$ of the microscopy-negative samples to apply to all other microscopy-negative samples, the overall prevalence and multiplicity by PCR were estimated.

\subsection{Data analysis}

Prospective analysis considered the time to the first fever episode, first clinical malaria and the first non-parasitaemic fever episode, using Kaplan-Meier analysis of times to episodes. The analysis was done using Epi-Info, version 6 (CDC, Atlanta, GA, USA) and SAS, version 8.2 (SAS Institute Inc., Cary, NC, USA).

\section{Results}

\subsection{Age-dependence of parasitological indices at baseline}

At baseline, $172 / 610(28 \%)$ of the children were positive for $P$. falciparum by microscopy, and 170 of these were confirmed positive by PCR. In addition, 15/88 microscopy-negative blood samples tested were found to be positive by PCR. By assuming this proportion to hold for the untested samples, we estimated the overall PCR positivity as $40 \%$. Geometric mean parasite density of microscopypositive samples was 1350.2 parasites/ $\mu \mathrm{l}(95 \% \mathrm{Cl}$ 1040.4-1752.3).

Parasite prevalence increased with age, whether assessed by microscopy or by PCR (Figure 1). The density of $P$. falciparum in microscopypositive slides peaked in children aged two years (Figure 2A).

The alleles of the marker gene msp2, can be grouped according to their central dimorphic domain into either the FC27 or the 3D7/Indochina 1 


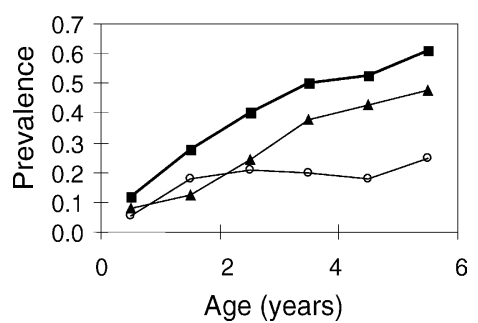

Figure 1. Prevalence of Plasmodium falciparum in Tanzanian children at baseline, by age. ( $\mathbf{\Delta}$ ) Proportion of slides positive by microscopy. ( $\bigcirc)$ Proportion of microscopynegative samples positive by PCR. (ם) Estimated overall prevalence by PCR.

allelic family. A total of 230 FC27 family infections and 291 3D7 family infections were detected in the samples positive by PCR at baseline while 3D7-type alleles accounted for $19 / 26$ of the infections found in samples negative by microscopy, but positive by $P C R$, suggesting that 3D7-type parasites tend to give infections with lower densities. This conclusion was supported by the finding that parasite density was correlated with the proportion of infecting alleles belonging to the FC27 family (Spearman's $r=0.19$, $P=0.05$ ).

Mean multiplicity of infection was 2.9 concurrent infections per carrier in samples positive by both microscopy and PCR. Mean multiplicity in microscopy-negative, but PCR-positive blood samples was 1.7 infections. These numbers led to the estimated overall mean multiplicity of 2.6 infections.

The parasite density was positively correlated with the overall multiplicity, with the strongest correlation in the youngest children and a steady decline in the correlation with age (Figure 2B). This pattern was accounted for by the effect of 3D7type infections. The correlation of densities with the FC27 multiplicity did not show a simple relationship with age (Figure 2B).
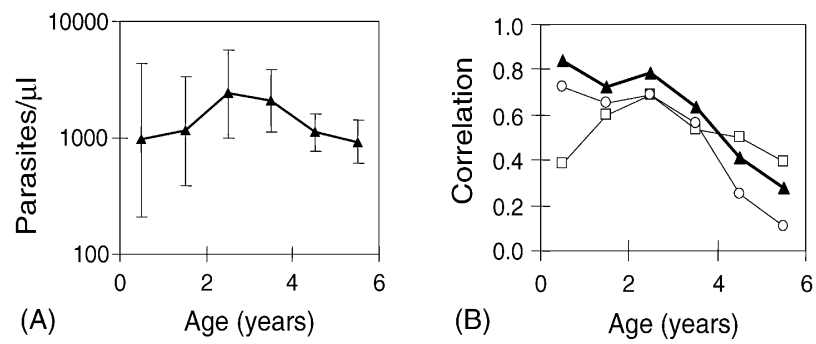

Figure 2. Age dependence and parasite densities in Tanzanian children. (A) Geometric mean parasite density $\pm 95 \% \mathrm{Cl}$. (B) Spearman's correlation between parasite density and multiplicity. ( $\Delta$ ) Overall multiplicity. ( $\square$ ) Multiplicity of FC27 infections. (O) Multiplicity of 3D7 infections.

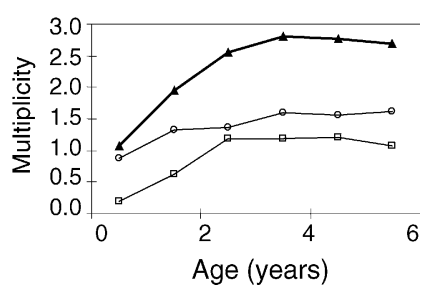

Figure 3. Multiplicity of Plasmodium falciparum infection in Tanzanian children, by age. ( $\mathbf{\Delta}$ ) Overall multiplicity. ( $\square$ ) Multiplicity of FC27 infections. ( $\bigcirc$ ) Multiplicity of 3D7 infections.

Multiplicity increased until the age of three years and after that remained stable (Figure 3). The increase was mainly accounted for by FC27 family parasites, which were infrequent in children aged less than two years (Figure 3 ).

\subsection{Morbidity follow-up by passive case detection}

Twelve of 610 children had a febrile episode at enrolment, and during the nine-month period of follow-up, 499 of these 610 children reported sick to the hospital or $\mathrm{MCH}$ clinic. Of these children, 70 presented subsequently with one or more clinical malaria episodes, but did not report with non-parasitaemic febrile illnesses. A further 169 presented with non-parasitaemic fevers but not with clinical malaria, and 46 experienced clinical malaria episodes and non-malaria fevers at different times.

The overall incidence of fevers decreased steeply with age; however, the incidence of $P$. falciparum positive fevers showed only a little agedependence, with a maximum in children aged two years. Consequently, fever episodes without concomitant parasitaemia predominated in those children who were aged less than two years at baseline, while parasitaemic episodes were relatively frequent in older children (Figure 4).

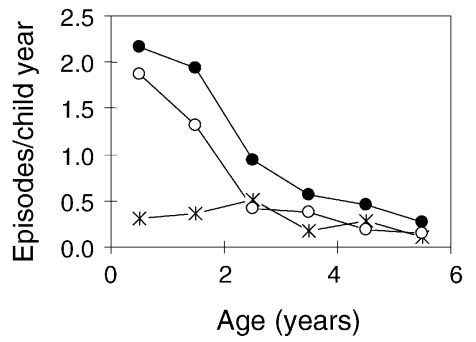

Figure 4. Incidence of fever in Tanzanian children, by age. (O) All fevers. ( $\bigcirc)$ Non-malaria fevers. (*) Clinical malaria. 
The main objective of the analysis was to examine how the incidence of clinical malaria depended on the parasitological status at baseline. The effects varied with the age of the children. In the youngest age groups, malaria parasites at baseline were a risk factor for subsequent clinical attacks, while in older children, baseline parasitaemia appeared to be a protective factor, so that children aged more than three years who were parasite-negative at baseline were more likely to report clinical malaria episodes than their initially parasite-positive contemporaries (log-rank test of the effect of positivity on survival times, adjusted for age, $\chi^{2}=1$, d.f. $=0.7, P=0.4$; interaction between age and positivity effect, $\chi^{2}=1$, d.f. $=11.5$, $P<0.001)$. This strong age trend in the relative risk associated with parasitaemia at baseline (Figure 5) was not seen for aparasitaemic fever episodes. Parasitaemia at baseline was also associated with reduced risk of non-parasitaemic fevers in all but the youngest children.

The risk associated with multiple infections at baseline was compared with that of single clone infections, to test whether the diversity of coinfecting parasites would modify the degree of protection or risk associated with infection per se (Table 1). In children aged less than three years, there was a tendency for multiple infections to be associated with higher prospective risk of clinical malaria, but in children aged more than three years, the tendency was for multiple infections to appear to protect against clinical malaria. However, none of the effects of multiplicity on clinical malaria incidence were statistically significant. Similarly, there were no significant effects of the allelic families of infecting parasites on the risk of subsequent clinical malaria when the potentially confounding effects of the age of the host were allowed for (data not shown).

In contrast to the effects on clinical malaria episodes, children aged less than three years with

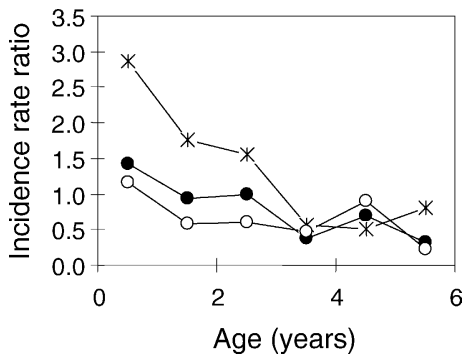

Figure 5. Effect of Plasmodium falciparum positivity at baseline on incidence of fever in Tanzanian children. ( $(\mathbf{O})$ All fevers. $(\bigcirc)$ Non-malaria fevers. $(*)$ Clinical malaria. multiple infections had less risk of non-malaria fevers than singly infected children in the same age group, but this effect was not evident in older children (log-rank $x^{2}=1$, d.f. $=4.9, P=0.02$ for the overall effect of multiple infections adjusted for age; $\chi^{2}=1$, d.f. $=13.7, P<0.001$ testing whether this effect is age dependent).

\subsection{Characteristics of clinical malaria episodes}

Two hundred and forty-seven blood samples from febrile patients, collected on attendance at the passive case detection facilities, were analysed by PCR. Ninety-six of these were positive and genotyped at the msp2 locus. Forty-four of the positive samples contained only 3D7 alleles, 43 mixed family infections and 9 FC27-type parasites only. The densities in the FC27-type infections were higher than in infections by both families or by 3D7 alleles only (FC27 family, geometric mean 7631 parasites/ $\mu$ l, 95\% Cl 460-125 000; 3D7 family, geometric mean 534 parasites/ $\mu \mathrm{l}$, 95\% Cl 135-2105; mixed infections, geometric mean 788 parasites $/ \mu \mathrm{l}, 95 \% \mathrm{Cl}$ 185-3362). The number of children from whom parasites were typed both at baseline and from a sample collected from a subsequent clinical episode was too small to allow any definite conclusions regarding the effect of baseline genotypes on the genotypes of parasites when the individual became sick.

\section{Discussion}

This study was designed to test whether infection status at baseline would determine the subsequent malaria incidence and how the acquisition of clinical immunity to malaria (and hence the age of the host) modifies this relationship. Our earlier suggestion was that in semi-immune individuals exposed to continual infection, $P$. falciparum infections stimulate short-term cross-protective responses, and, hence, hosts with multiple infections are at relatively low risk of subsequent morbidity. Conversely, in groups with little previous exposure, we suggested that malaria infection, and in particular high multiplicity of infection, is a marker of recent inoculations. These hosts, therefore, have higher risk of further infections and clinical attacks (Smith et al., 1999b).

This hypothesis is difficult to test because correlations between clinical episodes and infection status can easily arise as a result of confounding factors such as heterogeneous use of health 


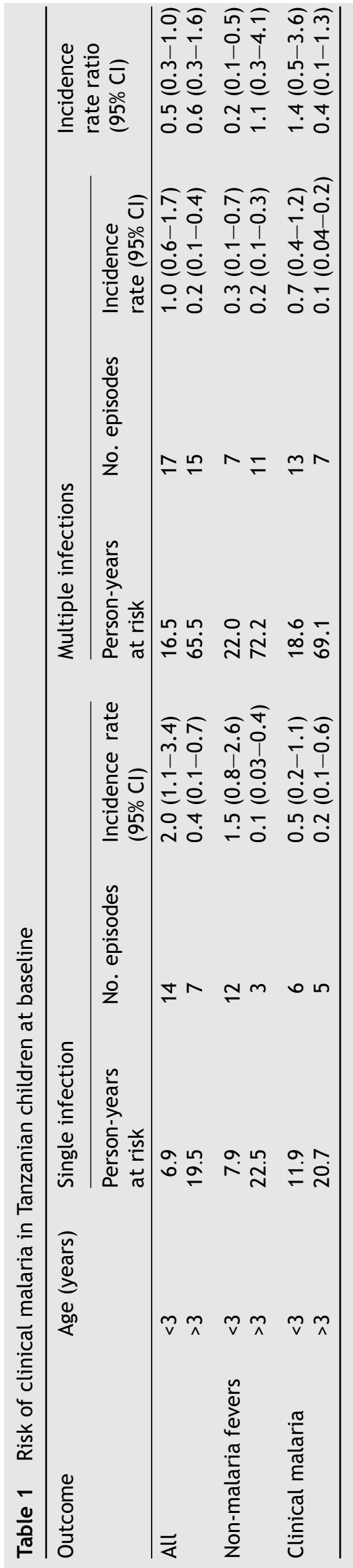

facilities (Branch et al., 2001), or host genetic polymorphisms that affect the host response to infection. Therefore, we designed our study to compare different age groups within the same population. Since it is unlikely that the effects of such confounding factors are age dependent, the differences with age in the relationships between risk of clinical attacks and parasitological status are most likely due to effects of acquired immunity.

The results were broadly consistent with previous findings from Kilombero valley, in that preexisting infections seemed to confer some protection against clinical episodes in the older, but not the younger children, and the correlation between parasite density and multiplicity (which also indicates the degree of interaction between clones) decreases with the age of the host. However, the level of $P$. falciparum endemicity in Ifakara town proved to be much lower than had been expected on the basis of previous studies (Charlwood et al., 1998; Smith et al., 1993). It is now apparent that, owing to ongoing malaria control activities and urbanization, there has been a sustained decrease in transmission in this area in the last decade (Schellenberg et al., 2003). A recent study reported an EIR of as low as 29 (95\% Cl 19-44) bites per annum (Drakeley et al., 2003). As a consequence of the lower transmission rate our study had less power than expected and even the oldest children in our study had experienced much less $P$. falciparum exposure than those children elsewhere in whom multiple infections appeared to be protective (Al Yaman et al., 1997a; Beck et al., 1997; Contamin et al., 1996; Farnert et al., 1999; Robert et al., 1996). The mean multiplicity in our study was lower than in most of these studies. The children in the older age group with multiple infections were too few for it to be possible to demonstrate whether the multiplicity conferred any significant protection.

The different contributions of the two allelic families of $m s p 2$, both to the load of asymptomatic infections and to the clinical episodes also broadly supported findings from other studies. In particular, the increase in the proportion of FC27 family infections with age and the higher parasite densities associated with this family confirm the previous findings from both Papua New Guinea (Felger et al., 1994) and Tanzania (Beck et al., 1999), which suggested that the alleles of the FC27 family were more successful in evading the host's immune response. In support of this, both a case control study, and a vaccine field trial in Papua New Guinea found that FC27-type alleles were strongly associated with malaria morbidity (Engelbrecht et 
al., 1995; Genton et al., 2002). Another study at the same site found that asymptomatic 3D7 family infections appeared to protect against subsequent morbidity, but FC27 family infections did not (Al Yaman et al., 1997b). In contrast, a study in Senegal failed to find any association between msp2 type and disease severity (Robert et al., 1996).

As a possible mechanism underlying such associations with morbidity, Engelbrecht et al. (1995) suggested that epitopes from FC27-type MSP2 might elicit strong but less protective antibody responses (the 'smokescreen' hypothesis), thus enabling increased proliferation and higher densities of parasites with a FC27-type MSP2.

Several other studies have found apparent protection conferred by $P$. falciparum infections against non-malaria fevers (e.g. Muller et al., 2001), but others did not find such protection (Al Yaman et al., 1997a). These discrepancies lend themselves to several possible interpretations. Malaria infections may genuinely reduce susceptibility to non-malaria fevers by modulating nonspecific immune responses. However, non-malaria fevers will be under-recorded in initially parasitaemic individuals because infecting malaria parasites may persist until the next febrile episode, which would then be misclassified as a clinical malaria episode, rather than a non-malaria episode. Children with asymptomatic infections may also represent a group of under-users of health facilities, either for socio-economic reasons or genetic predisposition to experience fewer symptoms. This too could lead to reduced attendance with non-malaria fevers in those children with parasites at baseline. These effects are likely to vary in importance depending on malaria endemicity and on the nature of the morbidity surveillance and health system.

None of these explanations contraindicate the treatment of asymptomatic parasitaemia in very young children, since there is no indication of any protective effect in the youngest age groups. This has the practical consequence of supporting the use of chemoprophylaxis and of intermittent treatment in young children in areas like Ifakara town. Randomized controlled trials of maloprim prophylaxis in infants (Menendez et al., 1997) and of intermittent treatment of infants (Schellenberg et al., 2001) have already shown high efficacy and substantial benefits that will now be evaluated for their effectiveness.

However, we would advocate caution in introducing such measures to older children as clearance of asymptomatic parasitaemia in older children may, in at least some settings, increase their subsequent risk of clinical malaria.

\section{Conflicts of interest statement}

For the period when this study was conducted and analysed, the authors have no conflicts of interest concerning the work reported in this paper.

\section{Acknowledgements}

This work was supported by the Swiss National Science Foundation (grants no. 31-52967.97 and 3152984.97) and the Daniela and Jürgen Westphal Stiftung. We thank C. Spycher and A. Schaub for their help in genotyping, the staff of IHRDC for assistance in microscopy and data entry and the medical personnel of the SFDDH together with C.M. Galindo and C.J. Acosta for supporting us in field surveys and case detection.

\section{References}

Al Yaman, F., Genton, B., Reeder, J.C., Anders, R.F., Smith, T., Alpers, M.P., 1997a. Reduced risk of clinical malaria in children infected with multiple clones of Plasmodium falciparum in a highly endemic area: a prospective community study. Trans. R. Soc. Trop. Med. Hyg. 91, 602-605.

Al Yaman, F., Genton, B., Reeder, J.C., Mokela, D., Anders, R.F., Alpers, M.P., 1997b. Humoral response to defined Plasmodium falciparum antigens in cerebral and uncomplicated malaria and their relationship to parasite genotype. Am. J. Trop. Med. Hyg. 56, 430-435.

Alonso, P.L., Smith, T., Schellenberg, J.R., Masanja, H., Mwankusye, S., Urassa, H., Bastos de Azevedo, I., Chongela, J., Kobero, S., Menendez, C., 1994. Randomised trial of efficacy of SPf66 vaccine against Plasmodium falciparum malaria in children in southern Tanzania. Lancet. 344, 11751181.

Arnot, D., 1998. Unstable malaria in Sudan: the influence of the dry season. Clone multiplicity of Plasmodium falciparum infections in individuals exposed to variable levels of disease transmission. Trans. R. Soc. Trop. Med. Hyg. 92, 580-585.

Beck, H.P., Felger, I., Huber, W., Steiger, S., Smith, T., Weiss, N., Alonso, P., Tanner, M., 1997. Analysis of multiple Plasmodium falciparum infections in Tanzanian children during the phase III trial of the malaria vaccine SPf66. J. Infect. Dis. 175, 921-926.

Beck, H.P., Felger, I., Vounatsou, P., Hirt, R., Tanner, M., Alonso, P., Menendez, C., 1999. Effect of iron supplementation and malaria prophylaxis in infants on Plasmodium falciparum genotypes and multiplicity of infection. Trans. R. Soc. Trop. Med. Hyg. 93 (Suppl. 1), S/41-S/45.

Branch, O.H., Takala, S., Kariuki, S., Nahlen, B.L., Kolczak, M., Hawley, W., Lal, A.A., 2001. Plasmodium falciparum genotypes, low complexity of infection, and resistance to subsequent malaria in participants in the Asembo Bay Cohort Project. Infect. Immun. 69, 7783-7792.

Charlwood, J.D., Smith, T., Lyimo, E., Kitua, A.Y., Masanja, H., Booth, M., Alonso, P.L., Tanner, M., 1998. Incidence of Plasmodium falciparum infection in infants in relation to exposure to sporozoite-infected anophelines. Am. J. Trop. Med. Hyg. 59, 243-251. 
Contamin, H., Fandeur, T., Rogier, C., Bonnefoy, S., Konate, L., Trape, J.F., Mercereau-Puijalon, O., 1996. Different genetic characteristics of Plasmodium falciparum isolates collected during successive clinical malaria episodes in Senegalese children. Am. J. Trop. Med. Hyg. 54, 632-643.

Drakeley, C., Schellenberg, D., Kihonda, J., Sousa, C.A., Arez, A.P., Lopes, D., Lines, J., Mshinda, H., Lengeler, C., Armstrong Schellenberg, J., Tanner, M., Alonso, P., 2003. An estimation of the entomological inoculation rate for Ifakara: a semi-urban area in a region of intense malaria transmission in Tanzania. Trop. Med. Int. Health. 8, 767-774.

Engelbrecht, F., Felger, I., Genton, B., Alpers, M., Beck, H.P., 1995. Plasmodium falciparum: malaria morbidity is associated with specific merozoite surface antigen 2 genotypes. Exp. Parasitol. 81, 90-96.

Farnert, A., Rooth, I., Svensson, Snounou, G., Bjorkman, A., 1999. Complexity of Plasmodium falciparum infections is consistent over time and protects against clinical disease in Tanzanian children. J. Infect. Dis. 179, 989-995.

Felger, I., Tavul, L., Kabintik, S., Marshall, V., Genton, B., Alpers, M., Beck, H.P., 1994. Plasmodium falciparum: extensive polymorphism in merozoite surface antigen 2 alleles in an area with endemic malaria in Papua New Guinea. Exp. Parasitol. 79, 106-116.

Felger, I., Smith, T., Edoh, D., Kitua, A., Alonso, P., Tanner, M. Beck, H.P., 1999. Multiple Plasmodium falciparum infections in Tanzanian infants. Trans. R. Soc. Trop. Med. Hyg. 93 (Suppl. 1), $S / 29-S / 34$

Felger, I., Beck, H.P., 2002. Genotyping of Plasmodium falciparum. PCR-RFLP analysis. In: Doolan, D. (Ed.), Malaria Meth ods and Protocols. Methods in Molecular Medicine 72. Humana Press, Totawa, pp. 117-129.

Genton, B., Betuela, I., Felger, I., Al Yaman, F., Anders, R.F., Saul, A., Rare, L., Baisor, M., Lorry, K., Brown, G.V., Pye, D. Irving, D.O., Smith, T.A., Beck, H.P., Alpers, M.P., 2002. A recombinant blood-stage malaria vaccine reduces Plasmodium falciparum density and exerts selective pressure on parasite populations in a phase $1-2 b$ trial in Papua New Guinea. J. Infect. Dis. 185, 820-827.

Henning, L., Felger, I., Beck, H.P., 1999. Rapid DNA extraction for molecular epidemiological studies of malaria. Acta Trop. 72, 149-155.

Mayor, A., Saute, F., Aponte, J.J., Almeda, J., Gomez-Olive, F.X., Dgedge, M., Alonso, P.L., 2003. Plasmodium falciparum multiple infections in Mozambique, its relation to other malariological indices and to prospective risk of malaria morbidity. Trop. Med. Int. Health. 8, 3-11.

Menendez, C., Kahigwa, E., Hirt, R., Vounatsou, P., Aponte, J.J., Font, F., Acosta, C.J., Schellenberg, D.M., Galindo, C.M., Kimario, J., Urassa, H., Brabin, B., Smith, T.A., Kitua, A.Y., Tanner, M., Alonso, P.L., 1997. Randomised placebo-controlled trial of iron supplementation and malaria chemoprophylaxis for prevention of severe anaemia and malaria in Tanzanian infants. Lancet. 350, 844-850.

Muller, D.A., Charlwood, J.D., Felger, I., Ferreira, C., do Rosario, V., Smith, T., 2001. Prospective risk of morbidity in relation to multiplicity of infection with Plasmodium falciparum in Sao Tome. Acta Trop. 78, 155-162.

Ntoumi, F., Contamin, H., Rogier, C., Bonnefoy, S., Trape, J.F., Mercereau-Puijalon, O., 1995. Age-dependent carriage of multiple Plasmodium falciparum merozoite surface antigen-2 alleles in asymptomatic malaria infections 52,81 88.

Ofosu-Okyere, A., Mackinnon, M.J., Sowa, M.P., Koram, K.A., Nkrumah, F., Osei, Y.D., Hill, W.G., Wilson, M.D., Arnot, D.E., 2001. Novel Plasmodium falciparum clones and rising clone multiplicities are associated with the increase in malaria morbidity in Ghanaian children during the transition into the high transmission season. Parasitology 123, 113-123.

Robert, F., Ntoumi, F., Angel, G., Candito, D., Rogier, C., Fandeur, T., Sarthou, J.L., Mercereau-Puijalon, O., 1996. Extensive genetic diversity of Plasmodium falciparum isolates collected from patients with severe malaria in Dakar. Senegal. Trans. R. Soc. Trop. Med. Hyg. 90, 704-711.

Roper, C., Richardson, W., Elhassan, I.M., Giha, H., Hviid, L., Satti, G.M., Theander, T.G., Arnot, D.E., 1998. Seasonal changes in the Plasmodium falciparum population in individuals and their relationship to clinical malaria: a longitudinal study in a Sudanese village. Parasitology 116, 501-510.

Schellenberg, D., Menendez, C., Kahigwa, E., Aponte, J., Vidal, J., Tanner, M., Mshinda, H., Alonso, P., 2001. Intermittent treatment for malaria and anaemia control at time of routine vaccinations in Tanzanian infants: a randomised, placebocontrolled trial. Lancet. 357, 1471-1477.

Schellenberg, D., Aponte, J., Kahigwa, E., Mshinda, H., Tanner, M., Menendez, C., Alonso, P., 2003. The incidence in children of clinical malaria detected by active case detection in Ifakara, southern Tanzania. Trans. R. Soc. Trop. Med. Hyg. in press.

Smith, T., Charlwood, J.D., Kihonda, J., Mwankusye, S., Billingsley, P., Meuwissen, J., Lyimo, E., Takken, W., Teuscher, T. Tanner, M., 1993. Absence of seasonal variation in malaria parasitaemia in an area of intense seasonal transmission. Acta Trop. 54, 55-72.

Smith, T., Beck, H.P., Kitua, A., Mwankusye, S., Felger, I., FraserHurt, N., Irion, A., Alonso, P., Teuscher, T., Tanner, M., 1999a. Age dependence of the multiplicity of Plasmodium falciparum infections and of other malariological indices in an area of high endemicity. Trans. R. Soc. Trop. Med. Hyg. 93 (Suppl. 1), 15-20.

Smith, T., Felger, I., Tanner, M., Beck, H.P., 1999b. Premunition in Plasmodium falciparum infection: insights from the epidemiology of multiple infections. Trans. R. Soc. Trop. Med. Hyg. 93 (Suppl. 1), S/59-S/64.

Available online at www.sciencedirect.com

science@direct. 